\title{
CENTRAL SIMPLE ALGEBRAS WITH INVOLUTION
}

\author{
BY LOUIS HALLE ROWEN ${ }^{1}$
}

\author{
Communicated by Barbara L. Osofsky, February 23, 1977
}

We will carry the following hypotheses throughout this paper: $F$ is a field of characteristic $\neq 2 ; A$ is a central simple $F$-algebra, i.e. a simple $F$-algebra of finite dimension, with center $F ; A$ has an involution (*) of first kind, i.e. an antiautomorphism of degree 2 which fixes the elements of $F$. The classic reference on central simple algebras is [1], which also treats involutions.

The dimension of $A$ (over $F$ ) must be a perfect square, which we denote as $n^{2}$. A famous conjecture is that $A$ must be a tensor product of a matrix subalgebra (over $F$ ) and quaternion subalgebras (over $F$ ); since the conjecture is easily proved when $n<8$, the first case of interest is when $n=8$. The main theorem of this paper is the following result when $A$ is a division algebra.

MAIN THEOREM. If $n=8$, then $A$ has a maximal subfield which is a Galois extension over $F$, with Galois group $\mathbf{Z}_{2} \oplus \mathbf{Z}_{2} \oplus \mathbf{Z}_{2}$.

The proof relies heavily on a computational result of Rowen and Schild, which will be given below. Before sketching the proof of the main theorem, we start with some general results (true for any $n$ ), which can be verified easily.

Proposition 1. Given a subfield $K$ of $A$ containing $F$, we have an involution of $A$ (of the first kind), which fixes the elements of $K$.

Proposition 2. Suppose $A$ is also a division algebra. Suppose $K$ is a nonmaximal subfield of $A$ (containing $F$ ), with an automorphism $\varphi$ over $F$, having degree 2. Then $\varphi^{*}: K \rightarrow K^{*}$ can be given by conjugation in $A$, by an element which is symmetric (resp. antisymmetric) with respect to $(*)$.

Let $\bar{F}$ denote the algebraic closure of $F$, and let $M_{n}(\bar{F})$ be the algebra of matrices over $\bar{F}$. Then (*) induces an involution on $M_{n}(\bar{F}) \approx A \otimes_{F} \bar{F}$, given by $\left(\Sigma a_{i} \otimes \beta_{i}\right)^{*}=\Sigma a_{i}^{*} \otimes \beta_{i}$, for $a_{i} \in A$ and $\beta_{i} \in F$. We say (*) is of symplectic type if the extension of $(*)$ to $M_{n}(\bar{F})$ is symplectic, i.e. not cogredient to the transpose (of matrices), cf. [1, p. 155]. Such an involution exists iff $n$ is even, in which case we can build a "universal" $F$-algebra with symplectic type involu-

AMS (MOS) subject classifications (1970). Primary 16A40, 16A28, 16A38, 46K99; Secondary 16A04, 16A08.

Key words and phrases. Division ring, involution, symplectic type, maximal subfield, Galois extension, central simple algebra.

1 This work was supported in part by the Israel Committee for Basic Research. 
tion, which we call $F\left(Y, Y^{s}\right)$. (See [3, §5] for details of construction, and for the properties of $F\left(Y, Y^{s}\right)$; here we write " $F$ " in place of " $\Omega$ ", which is used in [3].) Let $F_{1}=$ Cent $F\left(Y, Y^{s}\right)$, which is a central simple $F_{1}$-algebra of dimension $n^{2}$, with symplectic-type involution. By [3, Theorem 30], we can prove our main theorem by showing (when $n=8$ ) that $F\left(Y, Y^{s}\right)$ has a maximal subfield which is a Galois extension over $F_{1}$, with Galois group $\mathbf{Z}_{2} \oplus \mathbf{Z}_{2} \oplus \mathbf{Z}_{2}$.

Henceforth set $n=8$. The key step to our main theorem is the following fact, which follows directly from a computation of Rowen and Schild [4] (done with the help of the IBM 370 computer at Bar Ilan University):

LEMMA 1. $F\left(Y, Y^{s}\right)$ has an element $x$ whose square is central.

(The element $x$ is in fact given by an explicit formula.) Now we sketch the proof of Theorem 1, working in $F\left(Y, Y^{s}\right)$. Using Proposition 1, we can find an involution (of first kind) under which $x$ is symmetric; using Proposition 2, we can then modify the involution (*) such that $(*)$ is symplectic and $x$ is antisymmetric; i.e. $x^{*}=-x$. Then there is a symmetric element $y$, such that $y x y^{-1}=$ $-x$. If $y^{2} \in F_{1}(x)$ then $y$ and $x$ generate a quaternion $F_{1}$-subalgebra invariant under (*); in such a case, one concludes that $F\left(Y, Y^{s}\right)$ is a tensor product of quaternion subalgebras, and the theorem follows immediately. Thus we may assume $F_{1}\left(y^{2}\right) \cap F_{1}(x)=F$. Note $F_{1}\left(y^{2}\right) \neq F_{1}(y)$, and $y$ (being symmetric) has degree 4 over $F_{1}$. Hence $\left[F_{1}\left(y^{2}\right): F\right]=2$. We can then find $z$ symmetric, such that $z x z^{-1}=x$ and $z y^{2} z^{-1}=-y^{2}+\operatorname{tr}\left(y^{2}\right) / 4$. If $z^{2} \in F_{1}\left(x, y^{2}\right)$ then $z^{2} \in F_{1}\left(y^{2}\right)$ (since $z^{2}$ is symmetric and $x, x y^{2}$ are antisymmetric); in this case $y^{2}$ and $z$ generate a quaternion subalgebra invariant under $(*)$, and again we are done. Thus, we may assume $F_{1}\left(z^{2}\right) \cap F_{1}\left(x, y^{2}\right)=0$. Hence $F_{1}\left(x, y^{2}, z^{2}\right)=$ $F_{1}(z) F_{1}\left(y^{2}\right) F_{1}\left(z^{2}\right)$, and the theorem follows immediately. Q.E.D.

One interesting aspect of this theorem is that $F\left(Y, Y^{s}\right)$ is used to produce a positive result. Previously, universal PI-algebras (without involution) had been used by Amitsur [2] to produce an important negative result.

\section{REFERENCES}

1. A. A. Albert, Structure of algebras, Amer. Math. Soc. Colloq. Publ., vol. 24, Amer. Math. Soc., Providence, R. I., 1961. MR 23 \#A912.

2. S. A. Amitsur, On central division algebras, Israel J. Math. 12 (1972), 408-420. MR 47 \#6763.

3. L. H. Rowen, Identities in algebras with involution, Israel J. Math. 20 (1975), 7095.

4. L. H. Rowen and U. Schild, A scalar expression for matrices with symplectic involution (to appear). ISRAEL

DEPARTMENT OF MATHEMATICS, BAR ILAN UNIVERSITY, RAMAT GAN, 